VOLUME 22 (2017) 1-10

DOI: $10.24330 /$ ieja.325913

\title{
BAER GROUP RINGS WITH INVOLUTION
}

\author{
Anil Khairnar and B. N. Waphare \\ Received: 15 April 2016; Revised: 19 May 2017 \\ Communicated by Abdullah Harmancı
}

\begin{abstract}
We prove that if a group ring $R G$ is a (quasi) Baer $*$-ring, then so is $R$, whereas converse is not true. Sufficient conditions are given so that for some finite cyclic groups $G$, if $R$ is (quasi-) Baer $*$-ring, then so is the group ring $R G$. We prove that if the group ring $R G$ is a Baer $*$-ring, then so is $R H$ for every subgroup $H$ of $G$. Also, we generalize results of Zhong Yi, Yiqiang Zhou (for (quasi-) Baer rings) and L. Zan, J. Chen (for principally quasi-Baer and principally projective rings).
\end{abstract}

Mathematics Subject Classification (2010): 16W10, 16S34

Keywords: Group ring, Baer $*$-ring, quasi-Baer $*$-ring

\section{Introduction}

A *-ring $R$ is a ring equipped with an involution $x \rightarrow x^{*}$, that is, an additive anti-automorphism of period at most two. Let $R$ be a $*$-ring. An element $e \in R$ is called a projection if it is self-adjoint (i.e. $e=e^{*}$ ) and idempotent (i.e. $e^{2}=e$ ). Let $S$ be a nonempty subset of $R$. We write $r_{R}(S)=\{a \in R \mid s a=0, \forall s \in S\}$, and is called the right annihilator of $S$ in $R$, and $l_{R}(S)=\{a \in R \mid$ as $=0, \forall s \in S\}$, is the left annihilator of $S$ in $R$.

In [9], Kaplansky introduced Baer rings and Baer $\star$-rings to abstract various properties of $A W^{*}$-algebras, von Neumann algebras and complete $*$-regular rings. Clark [6] introduced quasi-Baer rings as a generalization of Baer rings. He uses this condition to characterize a finite dimensional algebra with unity over an algebraically closed field which is isomorphic to a twisted matrix units semi-group algebra. A ring $R$ is said to be a Baer (respectively, quasi-Baer) ring if the left annihilator of any nonempty subset (respectively, any ideal) of $R$ is generated by an idempotent. A *-ring $R$ is said to be a Baer *-ring, if for every nonempty subset $S$ of $R, r_{R}(S)=e R$, where $e$ is a projection in $R$. A ring $R$ is called a right (left) p.p. (principally projective) ring, if for every $a \in R, r_{R}(a)=e R\left(l_{R}(a)=R e\right)$, for some

The first author gratefully acknowledges the University Grant Commission, New Delhi, India for the award of Teachers Fellowship under the faculty development program, during the $X I I^{t h}$ plan period (2012-2017). 
idempotent $e \in R$. A ring $R$ is called a p.p. ring (Rickart ring) if it is both right and left p.p. In [11], Waphare and Khairnar introduced multiplicatively finite elements in a ring. By restricting multiplicatively finite elements, Khairnar and Waphare [10] introduced generalized projections, a partial order on them and studied this poset in a Rickart $*$-ring. Birkenmeier [3] called a ring $R$ to be a right (left) principally quasi-Baer (p.q.-Baer) ring, if for every $a \in R, r_{R}(a R)=e R\left(l_{R}(R a)=R e\right)$, for some idempotent $e \in R$. A ring $R$ is called a p.q.-Baer ring if it is both right and left p.q.-Baer. In [4] Birkenmeier et al. introduced quasi-Baer $*$-rings, a $*$-ring $R$ is said to be a quasi-Baer $*$-ring, if for every right ideal $I$ of $R, r_{R}(I)=e R$, where $e$ is a projection in $R$. In the same paper [4], they provide examples of Baer rings which are quasi-Baer $*$-rings but not Baer $*$-rings.

The concept of Baer *-ring is naturally motivated by the study of functional analysis. For example, every von Neumann algebra is a Baer $*$-algebra. Herstein was convinced that the simplicity of the simple Lie algebra should follow solely from the fact that $R=M_{n}(F)$ ( $F$ a field) is a simple ring. This led him in the $1950^{\prime} s$ to develop his Lie theory of arbitrary simple rings with involutions. Early motivation for studying rings with involution (also called $\star$-rings) came from rings of operators. If $\mathscr{B}(H)$ is the set of all bounded linear operators on a (real or complex) Hilbert space $H$, then each $\phi \in \mathscr{B}(H)$ has an adjoint, $\operatorname{adj}(\phi) \in \mathscr{B}(H)$, and $\phi \rightarrow \operatorname{adj}(\phi)$ is an involution on the ring $\mathscr{B}(H)$.

A natural question for a given class of rings is, how does the given class behave with respect to polynomial extensions? In [1], Armendariz seems to be the first to consider the behavior of a polynomial ring over a Baer ring. Later, the extensions of such properties have been studied by several authors, Birkenmeier et al. [2,4] (quasi-Baer, quasi-Baer * and principally quasi-Baer property for polynomial and Laurent polynomial), Birkenmeier and Park [5] (quasi-Baer property for monoid ring), Hirano [8] (Baer and quasi-Baer property for monoid rings $R G$, where the monoid $G$ is an ordered monoid) and Groenewald [7] (Baer property for semigroup $\operatorname{ring} R G$, where $R$ is a reduced ring and $G$ is a u.p. (unique product) semigroup). The main idea in proving all these results is similar to that used in the cases of (Laurent) polynomial rings and it does not help for the group ring extension. Group rings are interesting algebraic structures and they play a central role in the representation theory of finite groups. More recently, some applications in algebraic coding theory were given.

In the Open Problem Section of the Third International Symposium on Ring Theory (Kyongju, South Korea, 1999), Hirano asked the question, "Whether the group ring $R G$ is quasi-Baer if $R$ is quasi-Baer and $G$ is a finite group with $|G|^{-1} \epsilon$ 
$R$ ?". Zhong Yi and Yiqiang Zhou [12] answer the Hirano's question. In [13,14], L. Zan and J. Chen discuss the similar question for a p.p. and p.q.-Baer rings.

The objective of this paper is to answer the question, "Whether the group ring $R G$ is a (quasi-) Baer $*$-ring if $R$ is a (quasi) Baer $*$-ring and $G$ is a finite group with $|G|^{-1} \in R$ ?". We prove that, if the group ring $R G$ is a (quasi-) Baer $*$-ring, then so is $R$. Examples of a (quasi-) Baer $*$-ring $R$ and a finite group $G$ such that $|G|^{-1} \in R$ but the group ring $R G$ is not a (quasi-) Baer $*$-ring are given.

The following results are proved for a ring $R \subseteq \mathbb{C}$ (set of complex numbers) in $[12,13,14]$.

Lemma 1.1 ([12], Lemma $3.5(2))$. If $R \subseteq \mathbb{C}$ be a ring with $3^{-1} \in R$, then $R C_{3} \cong$ $\frac{R[x]}{\left(x^{3}-1\right)} \cong \frac{R[x]}{(x-1)} \times \frac{R[x]}{\left(x^{2}+x+1\right)} \cong R \times \frac{R[x]}{\left(x^{2}+x+1\right)}$.

Proposition 1.2 ([14], Proposition 2.3). If $R \subseteq \mathbb{C}$ be a ring, then $R C_{3}$ is left p.q.-Baer if and only if $\frac{R[x]}{\left(x^{2}+x+1\right)}$ is left p.q.-Baer and $3^{-1} \in R$.

Proposition 1.3 ([13], Proposition 2.3). If $R \subseteq \mathbb{C}$ be a ring, then $R C_{3}$ is left p.p. if and only if $\frac{R[x]}{\left(x^{2}+x+1\right)}$ is left p.p. and $3^{-1} \in R$.

We obtain these results without assuming $R \subseteq \mathbb{C}$, by using similar algebraic techniques, and then obtain analogous results to rings with involution.

We give an example to show in general, ring isomorphisms need not be *isomorphisms. Therefore, whenever we have to prove two $*$-rings are $*$-isomorphic, we should give an explicit isomorphism that also preserves $*$.

Throughout in this paper, $R$ denotes an associative ring with identity and $G$ denotes an abelian group.

\section{Necessary condition}

Let $R$ be a $\star$-ring and $G$ be a group. The involution $*$ on $R$ can be naturally extended to an involution on a group ring $R G$, on a polynomial ring $R[x]$ and on a Laurent polynomial ring $R\left[x, x^{-1}\right]$ as follows.

Let $f=\sum_{g \in G} a_{g} g \in R G$, then $f^{*}=\sum_{g \in G} a_{g}^{*} g$. If $p(x)=\sum_{k=0}^{n} r_{k} x^{k} \in R[x]$, where $n$ is a non-negative integer, then $(p(x))^{*}=\sum_{k=0}^{n} r_{k}^{*} x^{k}$. Similarly for Laurent polynomial ring $R\left[x, x^{-1}\right]$. If $S$ is a ring with involution $\bar{*}$, define an involution $\diamond$ on the product $R \times S$; for $(r, s) \in R \times S$ by $(r, s)^{\diamond}=\left(r^{*}, s^{\bar{*}}\right)$.

Remark 2.1. An ideal $I$ of $a *$-ring $R$ is said to be $a *$-ideal, if it is closed under involution; that is $x \in I$ implies $x^{*} \in I$. Let $R$ be $a *$-ring, $I$ be $a *$-ideal of $R$. We extend an involution * of $R$ to the quotient ring $R / I$. For $(a+I) \in R / I$, 
$(a+I)^{\diamond}=a^{*}+I$. Clearly $\diamond$ is an involution on the ring $R / I$ and hence $R / I$ is a ring with involution $\diamond$.

We denote all involutions by $*$, since it will not lead to ambiguity. We start by proving the following theorem.

Theorem 2.2. Let $R$ be $a *$-subring of $a *$-ring $S$ such that both share the same identity. Suppose that $S$ is a free left $R$-module with a basis $B$ such that $1 \in B$ and $a g=$ ga for all $a \in R$ and $g \in B$. If $S$ is a (quasi-) Baer $*$-ring, then so is $R$.

Proof. Let $I$ be a right ideal of $R$. Then $I S$ is a right ideal of $S$. Since $S$ is a quasiBaer $*$-ring, $r_{S}(I S)=e S$ for some $e^{2}=e^{*}=e \in S$. Let $e=e_{0} 1+e_{1} g_{1}+e_{2} g_{2}+\cdots+e_{n} g_{n}$, where $e_{i} \in R$ and $1=g_{0}, g_{i} \in B$. Let $b \in I$. For all $r \in R, 0=b r e=b r\left(e_{0} 1+e_{1} g_{1}+\right.$ $\left.e_{2} g_{2}+\cdots+e_{n} g_{n}\right)=$ bre $_{0} 1+$ bre $_{1} g_{1}+\cdots+$ bre $_{n} g_{n}$. Thus bre $e_{i}=0$ for $i=0,1, \cdots, n$ and for all $r \in R$ and $b \in I$. This yields $I R e_{i}=\{0\}$ for $i=0,1, \cdots, n$, therefore $I S e_{i}=I\left(\oplus_{g \in B} R g\right) e_{i}=\left(\sum_{g \in B}(I R) g\right) e_{i}=\sum_{g \in B}\left(I R e_{i}\right) g=\{0\}$ for $i=0,1, \cdots, n$. So $e_{i} \in r_{S}(I S)=e S \Rightarrow e_{i}=e e_{i}$ for $i=0,1, \cdots, n$. In particular $e_{0}=e e_{0}$, that is $e_{0}=e_{0}^{2} 1+e_{1} e_{0} g_{1}+\cdots+e_{n} e_{0} g_{n} \Rightarrow e_{0}^{2}=e_{0}$. Since $g_{i}^{*} \neq 1$ for any $i=1,2, \cdots, n, 1^{*}=1$ and $e^{*}=e$, we have $e_{0}^{*}=e_{0}$. Since $I R e_{i}=\{0\}$ for $i=0,1, \cdots, n$, so $I R e_{0}=\{0\} \Rightarrow e_{0} \epsilon$ $r_{R}(I) \Rightarrow e_{0} R \subseteq r_{R}(I)$.

Let $x \in r_{R}(I)$, so $I x=\{0\}$. Consider $I S x=I\left(\oplus_{g \in B} R g\right) x=\left(\sum_{g \in B}(I R) g\right) x=$ $\sum_{g \in B}(I R x) g=\{0\}$. This yields $x \in r_{S}(I S)=e S$, hence $x=e x=\left(e_{0} 1+e_{1} g_{1}+e_{2} g_{2}+\right.$ $\left.\cdots+e_{n} g_{n}\right) x=e_{0} 1 x+e_{1} g_{1} x+e_{2} g_{2} x+\cdots+e_{n} g_{n} x=e_{0} x 1+e_{1} x g_{1}+e_{2} x g_{2}+\cdots+e_{n} x g_{n}$. So $x=e_{0} x \Rightarrow x \in e_{0} R$. Hence $r_{R}(I R)=e_{0} R$. Therefore $R$ is a quasi-Baer $*$-ring. Similarly, we can prove if $S$ is a Baer $*$-ring, then so is $R$.

Corollary 2.3. Let $R$ be $a *$-ring and $G$ be a group. If the group ring $R G$ is a (quasi-) Baer *-ring, then so is $R$.

Proof. The proof follows from Theorem 2.2 by considering $S=R G=\oplus_{g \in G} R g$ as a free right $R$-module with basis $G$.

Corollary 2.4. If the polynomial ring $R[x]$ or the Laurent polynomial ring $R\left[x, x^{-1}\right]$ is a (quasi-) Baer*-ring, then so is $R$.

Proof. The proof follows from Theorem 2.2 by taking into account the basis $\left\{x^{i} \mid i=0,1,2, \cdots\right\}$ of $R[x]$ and $\left\{x^{i} \mid i=0, \pm 1, \pm 2, \cdots\right\}$ of $R\left[x, x^{-1}\right]$ over $R$.

If $R_{1}$ and $R_{2}$ are rings with involutions $\star_{1}$ and $\star_{2}$ respectively; $R_{1}$ and $R_{2}$ are said to be $*$-isomorphic if there is a ring isomorphism $\phi: R_{1} \rightarrow R_{2}$ such that $\phi\left(r^{*_{1}}\right)=(\phi(r))^{*_{2}}$ for all $r \in R_{1}$, in this case, we write $R_{1} \cong{ }^{*} R_{2}$. The following 
example shows that in general, a ring isomorphism need not be a $*$-isomorphism. An involution $*$ of a ring $R$ is said to be proper, if for $a \in R, a^{*} a=0$ implies $a=0$.

Example 2.5. Let $R_{1}=\mathbb{Z}_{2} \times \mathbb{Z}_{2}$ with the identity involution $*_{1}$ and $R_{2}=\mathbb{Z}_{2} \times \mathbb{Z}_{2}$ with the involution $\star_{2}: R_{2} \longrightarrow R_{2}$ given by $*_{2}((a, b))=(b, a)$. Define $f: R_{1} \rightarrow R_{2}$ by $f((a, b))=(a, b)$, then $f$ is a ring isomorphism but not $a *$-isomorphism. In fact, there does not exist $a *$-isomorphism between $R_{1}$ and $R_{2}$, as involution on $R_{1}$ is proper where as involution on $R_{2}$ is not proper.

Corollary 2.6. Let $R$ be $a *$-ring. If $R[x] /\left(x^{n}+a_{1} x^{n-1}+\cdots+a_{n}\right)$ is a (quasi-) Baer $*$-ring, where $a_{1}, a_{2}, \cdots, a_{n} \in R$ and $n$ is a positive integer, then $R$ is a (quasi-) Baer *-ring.

Proof. Observe that $S=R[x] /\left(x^{n}+a_{1} x^{n-1}+\cdots+a_{n}\right) \cong^{*} \oplus_{i=0}^{n-1} R x^{i}$ is a free left $R$-module with a basis $\left\{1, x, x^{2}, \cdots, x^{n-1}\right\}$ satisfying the assumptions of Theorem 2.2 .

Corollary 2.7. If $R[x] /\left(x^{n}+a_{1} x^{n-1}+\cdots+a_{n}\right)$ is a (quasi-) Baer ring, where $a_{1}, a_{2}, \cdots, a_{n} \in R$ and $n$ is a positive integer, then $R$ is a (quasi-) Baer ring.

Proof. Similar to the proof of Corollary 2.6 and using [12, Theorem 2.1].

The following result is essentially due to Zhong Yi and Yiqiang Zhou [12].

Theorem 2.8. Let $R$ be $a *$-ring and $G$ be a finite group. If the group ring $R G$ is a quasi-Baer *-ring, then $R$ is a quasi-Baer *-ring and $|G|^{-1} \in R$.

Proof. By Corollary 2.3, $R$ is a quasi-Baer $*$-ring. Since every quasi-Baer $\star$-ring is a left quasi-Baer ring, by [12, Theorem 2.4], $|G|^{-1} \in R$.

As an application of Theorem 2.8, we have the following example, which shows that the converse of Corollary 2.3 is not true in general.

Example 2.9. The ring of integers $\mathbb{Z}$ is an integral domain, so it is Baer *-ring with identity involution $*$ on $\mathbb{Z}$. By Theorem $2.8, \mathbb{Z} G$ is not a quasi-Baer $*$-ring for any nontrivial finite group $G$.

\section{3. (Quasi-) Baer group ring $R G$ with involution, where $G$ is a finite cyclic group}

For a positive integer $n, C_{n}$ denote the cyclic group of order $n$. From the following result, we conclude that for the group $G=C_{2}$, the converse of Theorem 2.8 is true.

Lemma 3.1. Let $R$ be $a *$-ring. If $2^{-1} \in R$, then $R C_{2} \cong^{*} R \times R$. 
Proof. Let $C_{2}=\{1, g\}$, define $f: R C_{2} \rightarrow R \times R$ by $f(a+b g)=(a+b, a-b)$, by [12, Lemma 3.1], $f$ is a ring isomorphism. Consider $f\left((a+b g)^{*}\right)=f\left(\left(a^{*}+b^{*} g\right)\right)=$ $\left(a^{*}+b^{*}, a^{*}-b^{*}\right)=(a+b, a-b)^{*}=(f(a, b))^{*}$. Hence $f$ is a $*$-isomorphism. Thus $R C_{2} \cong \cong^{*} R \times R$.

Proposition 3.2. $R C_{2}$ is a (quasi-) Baer $*$-ring if and only if $R$ is a (quasi-) Baer *-ring and $2^{-1} \in R$.

Proof. If $R C_{2}$ is a (quasi-) Baer $*$-ring, then by Theorem $2.8, R$ is a (quasi-) Baer $\star$-ring and $2^{-1} \in R$. Conversely suppose $R$ is a (quasi-) Baer $*$-ring and $2^{-1} \in R$. By Lemma 3.1, $R C_{2} \cong^{*} R \times R$, hence $R C_{2}$ is a (quasi-) Baer $*$-ring.

The following example shows that the converse of the Theorem 2.8 is not true in general.

Example 3.3. Let $R_{0}=\left\{n / 2^{k} \mid n \in \mathbb{Z}, k\right.$ is a non-negative integer $\}, R=\{a+$ $\left.p b i \mid a, b \in R_{0}\right\}$, where $p>2$ is a prime. Observe that $R$ is a subring of $\mathbb{C}$ (complex numbers). Since $R$ is a commutative integral domain, it is a Baer *-ring with an identity involution *. Also $\frac{1}{4} \in R$ (hence $\frac{1}{2} \in R$ ). By [12, Example 3], $R C_{4}$ is not a quasi-Baer ring, hence it is not a quasi-Baer *-ring.

From the above example, we conclude that the converse of Theorem 2.8 is not true for the group $G=C_{4}$. Next, we try to put some extra condition on a (quasi-) Baer $*$-ring $R$, so that the group ring $R C_{4}$ becomes a (quasi-) Baer $*$-ring.

Lemma 3.4. Let $R$ be $a *$-ring. If $2^{-1} \in R$, then $R C_{4} \cong^{*} R \times R \times \frac{R[x]}{\left(x^{2}+1\right)}$.

Proof. Clearly $\left(x^{2}+1\right)$ is a $*$-ideal of $R[x]$ and hence by Remark $2.1, \frac{R[x]}{\left(x^{2}+1\right)}$ is a *-ring. Let $C_{4}=\left\{1, g, g^{2}, g^{3}\right\}$ and $e=\left(1+g^{2}\right) / 2$. Since $e$ is a central projection (as 2 is a self-adjoint element of $R$ ) of $R C_{4}, R C_{4} e$ and $R C_{4}(1-e)$ are $*$-rings. Clearly $R C_{4} \cong{ }^{*} R C_{4} e \times R C_{4}(1-e)$. Observe that $R C_{4} e=\{r e+s g e \mid r, s \in R\}$ and $R C_{4}(1-e)=$ $\{r(1-e)+s g(1-e) \mid r, s \in R\}$. Define $\phi: R C_{4} e \rightarrow \frac{R[x]}{\left(x^{2}-1\right)}$ by, $\phi(r e+s g e)=r+s \bar{x}$. By [12, Lemma 3.3], $\phi$ is a ring isomorphism. Let $z=r e+s g e \in R C_{4} e$, consider $\phi\left(z^{*}\right)=\phi\left((r e+s g e)^{*}\right)=\phi\left(e^{*} r^{*}+e^{*} s^{*} g\right)=\phi\left(r^{*} e+s^{*} g e\right)=r^{*}+s^{*} \bar{x}=(r+s \bar{x})^{*}=$ $(\phi(r e+s g e))^{*}=(\phi(z))^{*}$. Therefore $\phi$ is a $*$-isomorphism, hence $R C_{4} e \cong \cong^{*} \frac{R[x]}{\left(x^{2}-1\right)}$. Similarly $R C_{4}(1-e) \cong \frac{R[x]}{\left(x^{2}+1\right)}$. Note that $R C_{2} \cong^{*} \frac{R[x]}{\left(x^{2}-1\right)}$ with a *-isomorphism $\psi: R C_{2} \rightarrow \frac{R[x]}{\left(x^{2}-1\right)}$ define by $\psi(r 1+s g)=r+s \bar{x}$. By Lemma 3.1, $R C_{2} \cong^{*} R \times R$. In nutshell, we have $R C_{4} \cong *$ 药 $R \times \frac{R[x]}{\left(x^{2}+1\right)}$.

Note: If $R$ is a quasi-Baer ring ( $*$-ring) and $e$ is a central idempotent (projection) in $R$, then $e R e=R e=e R$ is a quasi-Baer ring (*-ring). Similar result is true for Baer and Baer $*$-rings. 
Proposition 3.5. $R C_{4}$ is a (quasi-) Baer *-ring if and only if $\frac{R[x]}{\left(x^{2}+1\right)}$ is a (quasi-) Baer $*$-ring and $2^{-1} \in R$.

Proof. If $R C_{4}$ is a (quasi-) Baer $*$-ring, then by Theorem $2.8, R$ is a (quasi-) Baer *-ring and $2^{-1} \in R$. By Lemma $3.4, R C_{4}(1-e) \cong \cong^{*} \frac{R[x]}{\left(x^{2}+1\right)}$. As $(1-e)$ is a central projection, $R C_{4}(1-e)$ is a (quasi-) Baer $*$-ring. Hence $\frac{R[x]}{\left(x^{2}+1\right)}$ is a (quasi-) Baer $*-$ ring. Conversely suppose $\frac{R[x]}{\left(x^{2}+1\right)}$ is a (quasi-) Baer $*$-ring and $2^{-1} \in R$, by Corollary 2.6, $R$ is a (quasi-) Baer $*$-ring. Since $2^{-1} \in R$, by Lemma 3.4, $R C_{4} \cong^{*} R \times R \times \frac{R[x]}{\left(x^{2}+1\right)}$, hence $R C_{4}$ is a (quasi-) Baer $*$-ring.

The remaining section is devoted to discuss the situation for $G=C_{3}$. For the group $G=C_{3}$, in $[12,13,14]$ the situation is discussed for the rings $R \subseteq \mathbb{C}$ in the context of quasi-Baer, p.q.-Baer and p.p. rings. We generalized these results to any ring $R$. Further, we prove an analogue of these results to (quasi-) Baer $*$-rings.

Example 3.6. Let $R_{0}=\left\{n / 6^{k} \mid n \in \mathbb{Z}, k\right.$ is a non-negative integer $\}, R=\{a+$ $\left.5 b \sqrt{3} i \mid a, b \in R_{0}\right\}$. Then $R$ is a subring of $\mathbb{C}$. Since $R$ is a commutative integral domain, it is a Baer *-ring with an identity involution *. Also $\frac{1}{3} \in R$. By $[12$, Example 4], $R C_{3}$ is not a quasi-Baer ring, hence it is not a quasi-Baer *-ring.

The following lemma can be obtained by using Chinese remainder theorem, but to prove $*$-isomorphism we explicitly give an isomorphism that preserves $*$.

Lemma 3.7. Let $R$ be a ring. If $3^{-1} \in R$, then $R C_{3} \cong R \times \frac{R[x]}{\left(x^{2}+x+1\right)}$.

Proof. First we prove $R C_{3} \cong \frac{R[x]}{\left(x^{3}-1\right)}$. A mapping $\phi: R C_{3} \rightarrow \frac{R[x]}{\left(x^{3}-1\right)}$ defined by $\phi\left(r_{1} 1+r_{2} g+r_{3} g^{2}\right)=r_{1}+r_{2} x+r_{3} \bar{x}$ is a ring isomorphism, hence $R C_{3} \cong \frac{R[x]}{\left(x^{3}-1\right)}$. Now we prove $\frac{R[x]}{\left(x^{3}-1\right)} \cong \frac{R[x]}{(x-1)} \times \frac{R[x]}{\left(x^{2}+x+1\right)}$. Let $I=\left(x^{3}-1\right), I_{1}=(x-1), I_{2}=\left(x^{2}+x+1\right)$, define $\psi$ : $\frac{R[x]}{I} \rightarrow \frac{R[x]}{I_{1}} \times \frac{R[x]}{I_{2}}$, by $\psi\left(r_{1}+r_{2} x+r_{3} x^{2}+I\right)=\left(\left(r_{1}+r_{2}+r_{3}\right)+I_{1},\left(r_{1}-r_{3}\right)+\left(r_{2}-r_{3}\right) x+I_{2}\right)$. It is easy to verify that $\psi$ is a ring homomorphism. To prove $\psi$ is one-one, suppose $\psi\left(r_{1}+r_{2} x+r_{3} x^{2}+I\right)=\psi\left(r_{1}^{\prime}+r_{2}^{\prime} x+r_{3}^{\prime} x^{2}+I\right)$, that is $\left(\left(r_{1}+r_{2}+r_{3}\right)+I_{1},\left(r_{1}-\right.\right.$ $\left.\left.r_{3}\right)+\left(r_{2}-r_{3}\right) x+I_{2}\right)=\left(\left(r_{1}^{\prime}+r_{2}^{\prime}+r_{3}^{\prime}\right)+I_{1},\left(r_{1}^{\prime}-r_{3}^{\prime}\right)+\left(r_{2}^{\prime}-r_{3}^{\prime}\right) x+I_{2}\right)$, this yields $\left(r_{1}+r_{2}+r_{3}\right)-\left(r_{1}^{\prime}+r_{2}^{\prime}+r_{3}^{\prime}\right) \in I_{1}$ and $\left(\left(r_{1}-r_{3}\right)-\left(r_{1}^{\prime}-r_{3}^{\prime}\right)\right)+\left(\left(r_{2}-r_{3}\right)-\left(r_{2}^{\prime}-r_{3}^{\prime}\right)\right) x \in I_{2}$. Hence $\left(r_{1}+r_{2}+r_{3}\right)-\left(r_{1}^{\prime}+r_{2}^{\prime}+r_{3}^{\prime}\right)=0,\left(r_{1}-r_{3}\right)-\left(r_{1}^{\prime}-r_{3}^{\prime}\right)=0$ and $\left(r_{2}-r_{3}\right)-\left(r_{2}^{\prime}-r_{3}^{\prime}\right)=0$. From this we have,

$$
\begin{gathered}
r_{1}+r_{2}+r_{3}=r_{1}^{\prime}+r_{2}^{\prime}+r_{3}^{\prime} \\
r_{1}-r_{3}=r_{1}^{\prime}-r_{3}^{\prime} \\
r_{2}-r_{3}=r_{2}^{\prime}-r_{3}^{\prime}
\end{gathered}
$$


$2-3 \Rightarrow r_{1}-r_{2}=r_{1}^{\prime}-r_{2}^{\prime} \Rightarrow r_{2}=r_{1}-r_{1}^{\prime}+r_{2}^{\prime}$. Put this value of $r_{2}$ in Equation 1, we get

$$
2 r_{1}+r_{3}=2 r_{1}^{\prime}+r_{3}^{\prime}
$$

$4+2 \Rightarrow 3 r_{1}=3 r_{1}^{\prime}$. Since $3^{-1} \in R$, we have $r_{1}=r_{1}^{\prime}$. So Equation $2 \Rightarrow r_{3}=$ $r_{3}^{\prime}$ and Equation $3 \Rightarrow r_{2}=r_{2}^{\prime}$, hence $r_{1}+r_{2} x+r_{3} x^{2}+I=r_{1}^{\prime}+r_{2}^{\prime} x+r_{3}^{\prime} x^{2}+I$. Therefore $\psi$ is a one-one mapping. Now we prove $\psi$ is an on-to mapping. Let $\left(a+I_{1}, b+c x+I_{2}\right) \in \frac{R[x]}{I_{1}} \times \frac{R[x]}{I_{2}}$. We have to find $r_{1}+r_{2} x+r_{3} x^{2}+I \in \frac{R[x]}{I}$ such that $\psi\left(r_{1}+r_{2} x+r_{3} x^{2}+I\right)=\left(\left(r_{1}+r_{2}+r_{3}\right)+I_{1},\left(r_{1}-r_{3}\right)+\left(r_{2}-r_{3}\right) x+I_{2}\right)=\left(a+I_{1}, b+c x+I_{2}\right)$. Consider equations $r_{1}+r_{2}+r_{3}=a, r_{1}-r_{3}=b$ and $r_{2}-r_{3}=c$. Using the fact $3^{-1} \in R$, we can solve these equations for $r_{1}, r_{2}$ and $r_{3}$ similarly as above. Therefore $\psi$ is an on-to mapping. Therefore $\psi$ is an isomorphism, hence $\frac{R[x]}{\left(x^{3}-1\right)} \cong \frac{R[x]}{(x-1)} \times \frac{R[x]}{\left(x^{2}+x+1\right)}$. It is clear that $\frac{R[x]}{(x-1)} \cong R$. In all we get $R C_{3} \cong R \times \frac{R[x]}{\left(x^{2}+x+1\right)}$.

Corollary 3.8 ([12], Lemma $3.5(2))$. If $R \subseteq \mathbb{C}$ be a ring with $3^{-1} \in R$, then $R C_{3} \cong \frac{R[x]}{\left(x^{3}-1\right)} \cong \frac{R[x]}{(x-1)} \times \frac{R[x]}{\left(x^{2}+x+1\right)} \cong R \times \frac{R[x]}{\left(x^{2}+x+1\right)}$.

Theorem 3.9. $R C_{3}$ is (quasi-) Baer if and only if $\frac{R[x]}{\left(x^{2}+x+1\right)}$ is quasi-Baer and $3^{-1} \in R$.

Proof. If $R C_{3}$ is (quasi-) Baer, then by [12, Corollary 2.2], $R$ is (quasi-) Baer and by [12, Theorem 2.4], $3^{-1} \in R$. By Lemma 3.7, $R C_{3} \cong R \times \frac{R[x]}{\left(x^{2}+x+1\right)}$. Observe that $\frac{R[x]}{\left(x^{2}+x+1\right)} \cong\left(R \times \frac{R[x]}{\left(x^{2}+x+1\right)}\right)\left(0,1+I_{2}\right)$, where $I_{2}=\left(x^{2}+x+1\right)$. As $\left(0,1+I_{2}\right)$ is a central idempotent, $\left(R \times \frac{R[x]}{\left(x^{2}+x+1\right)}\right)\left(0,1+I_{2}\right)$ is a (quasi-) Baer ring. Hence $\frac{R[x]}{\left(x^{2}+x+1\right)}$ is a (quasi-) Baer ring. Conversely suppose $\frac{R[x]}{\left(x^{2}+x+1\right)}$ is (quasi-) Baer and $3^{-1} \in R$. By Corollary 2.7, $R$ is a (quasi-) Baer ring. Therefore by Lemma $3.7, R C_{3}$ is a (quasi-) Baer ring.

The following result is a conclusion made by Zhong Yi and Yiqiang Zhou after Lemma 3.5 in [12].

Corollary 3.10. If $R \subseteq \mathbb{C}$ be a ring, then $R C_{3}$ is (quasi-) Baer if and only if $\frac{R[x]}{\left(x^{2}+x+1\right)}$ is (quasi-) Baer and $3^{-1} \in R$.

The proofs of Corollary 3.11 and Corollary 3.12 are similar to the proof of Corollary 3.10 .

Corollary 3.11 ([14], Proposition 2.3). If $R \subseteq \mathbb{C}$ be a ring, then $R C_{3}$ is left p.q.Baer if and only if $\frac{R[x]}{\left(x^{2}+x+1\right)}$ is left p.q.-Baer and $3^{-1} \in R$.

Corollary 3.12 ([13], Proposition 2.3). If $R \subseteq \mathbb{C}$ be a ring, then $R C_{3}$ is left p.p. if and only if $\frac{R[x]}{\left(x^{2}+x+1\right)}$ is left p.p. and $3^{-1} \in R$. 
Theorem 3.13. Let $R$ be $a *$-ring. If $3^{-1} \in R$, then $R C_{3} \cong^{*} R \times \frac{R[x]}{\left(x^{2}+x+1\right)}$.

Proof. Note that $\left(x^{3}-1\right)$ and $\left(x^{2}+x+1\right)$ are $*$-ideals of the ring $R[x]$. Hence by Remark 2.1, $\frac{R[x]}{\left(x^{3}-1\right)}$ and $\frac{R[x]}{\left(x^{2}+x+1\right)}$ are *-rings. Let $\phi$ and $\psi$ are mappings as in Lemma 3.7. Let $z=r_{1} 1+r_{2} g+r_{3} g^{2} \in R C_{3}$, consider $\phi\left(z^{*}\right)=\phi\left(r_{1}^{*} 1+r_{2}^{*} g+r_{3}^{*} g^{2}\right)=$ $r_{1}^{*}+r_{2}^{*} x+r_{3}^{*} \bar{x}=(\phi(z))^{*}$. Therefore $\phi$ is a $*$-isomorphism. To prove $\psi$ is $*$ preserving, consider $\psi\left(\left(r_{1}+r_{2} x+r_{3} x^{2}+I\right)^{*}\right)=\psi\left(r_{1}^{*}+r_{2}^{*} x+r_{3}^{*} x^{2}+I\right)=\left(\left(r_{1}^{*}+r_{2}^{*}+r_{3}^{*}\right)+I_{1},\left(r_{1}^{*}-r_{3}^{*}\right)+\right.$ $\left.\left(r_{2}^{*}-r_{3}^{*}\right) x+I_{2}\right)=\left(\left(r_{1}+r_{2}+r_{3}\right)+I_{1},\left(r_{1}-r_{3}\right)+\left(r_{2}-r_{3}\right) x+I_{2}\right)^{*}=\left(\psi\left(r_{1}+r_{2} x+r_{3} x^{2}+I\right)\right)^{*}$. Therefore $\psi$ is a $*$-isomorphism. As $\frac{R[x]}{(x-1)} \cong{ }^{*} R$, we have $R C_{3} \cong{ }^{*} R \times \frac{R[x]}{\left(x^{2}+x+1\right)}$.

Proposition 3.14. Let $R$ be $a *$-ring. $R C_{3}$ is a (quasi-) Baer *-ring if and only if $R[x] /\left(x^{2}+x+1\right)$ is a (quasi-) Baer *-ring and $3^{-1} \in R$.

Proof. If $R C_{3}$ is a (quasi-) Baer *-ring, then by Theorem 2.8, $R$ is a (quasi-) Baer *-ring and $3^{-1} \in R$. By Theorem 3.13, $R C_{3} \cong R \times \frac{R[x]}{\left(x^{2}+x+1\right)}$. Observe that $\frac{R[x]}{\left(x^{2}+x+1\right)} \cong{ }^{*}\left(R \times \frac{R[x]}{\left(x^{2}+x+1\right)}\right)\left(0,1+I_{2}\right)$, where $I_{2}=\left(x^{2}+x+1\right)$. As $\left(0,1+I_{2}\right)$ is a central projection, $\left(R \times \frac{R[x]}{\left(x^{2}+x+1\right)}\right)\left(0,1+I_{2}\right)$ is a (quasi-) Baer $*$-ring. Hence $\frac{R[x]}{\left(x^{2}+x+1\right)}$ is a (quasi-) Baer $*$-ring. Conversely suppose $R[x] /\left(x^{2}+x+1\right)$ is a (quasi-) Baer $*$-ring and $3^{-1} \in R$, so by Corollary $2.6, R$ is a (quasi-) Baer $*$-ring. Since $3^{-1} \in R$, by Lemma $3.7, R C_{3} \cong * \times R[x] /\left(x^{2}+x+1\right)$, hence $R C_{3}$ is a (quasi-) Baer $*$-ring.

Acknowledgment. The authors would like to thank the referee for the valuable suggestions and comments.

\section{References}

[1] E. P. Armendariz, A note on extensions of Baer and p.p.-rings, J. Austral. Math. Soc., 18 (1974), 470-473.

[2] G. F. Birkenmeier, J. Y. Kim and J. K. Park, On polynomial extensions of principally quasi-Baer rings, Kyungpook Math. J., 40(2) (2000), 247-253.

[3] G. F. Birkenmeier, J. Y. Kim and J. K. Park, Principally quasi-Baer rings, Comm. Algebra, 29(2) (2001), 639-660.

[4] G. F. Birkenmeier, J. Y. Kim and J. K. Park, Polynomial extensions of Baer and quasi-Baer rings, J. Pure Appl. Algebra, 159(1) (2001), 25-42.

[5] G. F. Birkenmeier and J. K. Park, Triangular matrix representations of ring extensions, J. Algebra, 265(2) (2003), 457-477.

[6] W. E. Clark, Twisted matrix units semigroup algebras, Duke Math. J., 34(3) (1967), 417-423.

[7] N. J. Groenewald, A note on extensions of Baer and p.p.-rings, Publ. Inst. Math. (Beograd) (N.S.), 34(48) (1983), 71-72. 
[8] Y. Hirano, On ordered monoid rings over a quasi-Baer ring, Comm. Algebra, 29(5) (2001), 2089-2095.

[9] I. Kaplansky, Rings of Operators, W. A. Benjamin, Inc., New YorkAmsterdam, 1968.

[10] A. Khairnar and B. N. Waphare, Order properties of generalized projections, Linear Multilinear Algebra, 65(7) (2017), 1446-1461.

[11] B. N. Waphare and A. Khairnar, Semi-Baer modules, J. Algebra Appl., 14(10) (2015), 1550145 (12 pp).

[12] Z. Yi and Y. Zhou, Baer and quasi-Baer properties of group rings, J. Aust. Math. Soc., 83(2) (2007), 285-296.

[13] L. Zan and J. Chen, p.p. properties of group rings, Int. Electron. J. Algebra, 3 (2008), 117-124.

[14] L. Zan and J. Chen, Principally quasi-Baer properties of group rings, Studia Sci. Math. Hungar., 49(4) (2012), 454-465.

Anil Khairnar (Corresponding Author)

Department of Mathematics

Abasaheb Garware College

Pune-411004, India

e-mail: anil.khairnar@mesagc.org; anil_maths2004@yahoo.com

\section{B. N. Waphare}

Center for Advanced Study in Mathematics

Department of Mathematics

Savitribai Phule Pune University

Pune-411007, India

e-mail: bnwaph@math.unipune.ac.in; waphare@yahoo.com 